\title{
PREFACE: MULTISCALE MODELLING OF MATERIALS AND STRUCTURES, PT. II
}

This special issue of the International Journal for Multiscale Computational Engineering is dedicated to symposium MS806, "Multiscale Modelling of Materials and Structures," organized within the ECCOMAS 2016 Congress on Crete Island. The issue contains ten papers selected from those presented during the symposium.

The European Congress on Computational Methods in Applied Sciences (ECCOMAS) brings together our scientific communities and identifies emerging research trends and issues. Such contacts are important for establishing cooperative research activities. The objective of the "Multiscale Modelling of Materials and Structures" symposium was to gather researchers working on various aspects of modelling of phenomena occurring in materials at various scales, from nano- to micro- and mezo- to macro scale, and to enable exchange of ideas and results.

A total of 28 papers were presented during the symposium, and after evaluation of the presentations, 12 authors were invited to submit extended versions of their papers to this special issue of the International Journal for Multiscale Computational Engineering (IJMCE). After the usual peer review procedure for the IJMCE, ten articles were accepted for publication. Five articles are published in the current issue, and the remaining five were published previously in issue 4.

These articles focus on topics connected with widely understood applications of multiscale methods, as well as atomistic and molecular static approaches. The papers deal with such multiscale modelling methods as finite elements and discrete elements for macro scale, as well as Cellular Automata, phase fields, and molecular simulations in lower dimensional scales. Subsequent papers describe such particular applications of multiscale modelling techniques as numerical determination of properties of composites, modelling of sintering, modelling of phase changes, analysis of ultrashort laser pulse treatment, dynamic analysis of metallic foams, and prediction of fractures in macro scale.

Beyond steels, the papers address a variety of advanced materials, including nonferrous metal alloys, graphenelike materials, titanium alloys, and reactive powder concrete. Two-phase microstructures are modelled using a phase-fieldbased solution of the diffusion equation. Finally, one paper discusses the meshless method in multiscale applications.

The guest editors of this issue express their thanks to the authors of the papers for their contributions. Particular thanks are directed to the reviewers for their hard work in reviewing the submitted papers and for help in maintaining the high publication standards of the IJMCE. It is our hope that this special issue of the International Journal for Multiscale Computational Engineering will be a source of inspiration for the readers in their scientific work.

\section{Guest Editors:}

Tadeusz Burczyński

Institute of Fundamental Technological Research

Polish Academy of Sciences

Warsaw, Poland

Xavier Oliver

Technical University of Catalonia

Barcelona, Spain
Maciej Pietrzyk

Agh University of Science and Technology

Krakow, Poland

Alfredo Huespe

Universidad Nacional del Litoral

Santa Fe, Argentina 